\title{
New proposals on water fluoridation offers fresh hope for nation's oral health
}

The Secretary of State Matt Hancock has confirmed plans for radical NHS reforms which include proposals to remove the barriers to water fluoridation schemes in the UK. The proposals are welcomed by the Oral Health Foundation who have been lobbying for water fluoridation to be extended for many years. If implemented this will help ease the process of water fluoridation for local communities and give the oral health of the nation a much-needed boost.

The addition of fluoride to water has been researched for over 75 years, and water fluoridation has been proven to reduce decay by $35 \%^{1}$. Fluoride can greatly help dental health by strengthening the tooth enamel, making it more resistant to tooth decay.

The draft bill, entitled 'Integration and Innovation: working together to improve health and social care for all', lays out plans for the government to take responsibility for the funding and implementation of water fluoridation, putting it in a position to drive forward and implement schemes.

Currently, some six million people in the UK receive a fluoridated water supply. Those in areas with fluoridated water have be shown to have lower rates of decay than those without.

At present decisions on water fluoridation sit within the powers of local authorities.

There has been little progress in expanding the UK's adoption of water fluoridation since the late 1980s.

Dr Nigel Carter OBE, Chief Executive of the Oral Health Foundation, said: 'In recent years we have seen water fluoridation proposals repeatedly stall at local authority level due to high cost, competing demands, and limited budgets. All the while, tooth

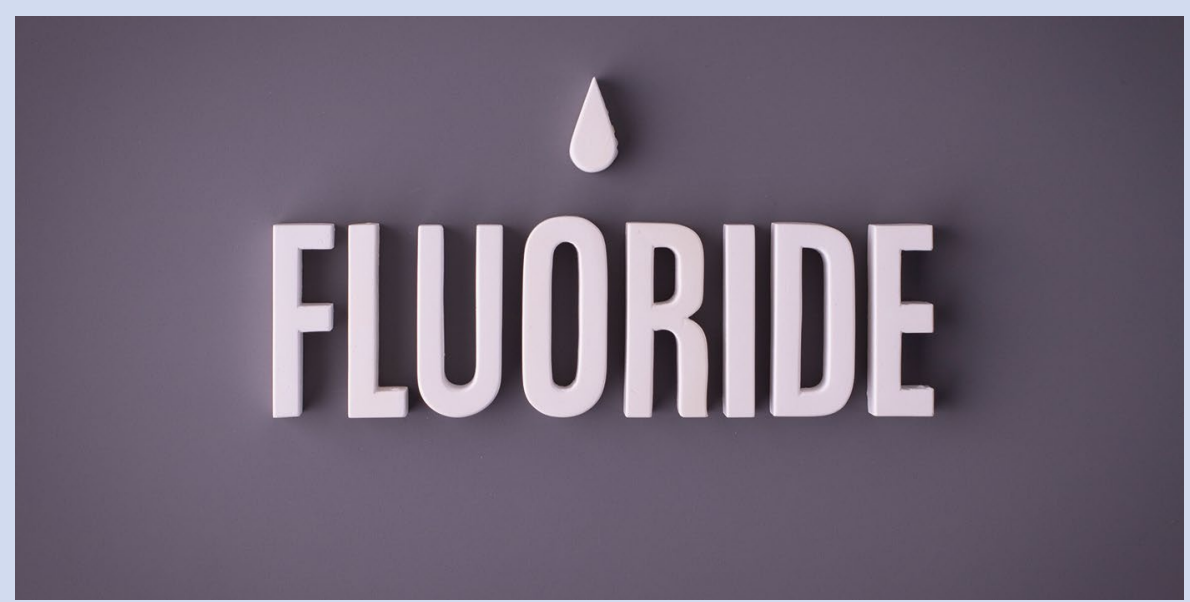

decay continues to be the most common chronic disease in the country.

'Children's oral health also continues to suffer. Tooth extractions, most of which caused by decay, remains the most common reason for hospital admissions of five-tonine year-olds in the UK. Yet this is a totally preventable disease.

'Tooth decay comes at a tremendous cost to the economy. Around two million people in the UK have taken time of work in the last five years due to poor oral health, at a cost to businesses of more than $£ 35 \mathrm{~m}$ a year.

'As a dental practitioner in Birmingham I saw first-hand the benefits to children's oral health of water fluoridation. Working on the borders of fluoridated Birmingham and the then non-fluoridated Sandwell we could tell which side of the dividing road children came from based solely on their decay experience.

'We believe that water fluoridation is the single most effective public health measure there is for reducing oral health inequalities and tooth decay rates, especially amongst children. We welcome these proposals and believe they represent an opportunity to take a big step forward in not only improving this generation's oral health, but those for decades to come.

\section{Reference}

1. Iheozor-Ejiofor Z, Worthington HV, Walsh T, et al. Water fluoridation for the prevention of dental caries. Cochrane Database of Systematic Reviews 2015; 6: CD010856.

\section{BDA NI welcomes $11.5 \mathrm{~m}$ boost to ventilation}

The British Dental Association Northern Ireland has welcomed the $£ 1.5$ million boost to dentistry announced today by the Department of Health, in response to our calls to support dental practices to invest in new infection prevention and control measures, including ventilation systems, to mitigate the impact of COVID-19 on dentistry.

Practices are open and safe - but most are running at a fraction of their former capacity, which means that thousands of patients are missing out on being seen by a dentist. The number one barrier that dentists face is the fallow time, the gap that has to be left between patients who require any procedures that generate aerosols (e.g. fillings or crowns), to minimise the risk of infection.

Many dentists can only bring that time down by investing in new ventilation equipment. The BDA welcomes funding to shorten the fallow time which will enable more patients to be seen by dentists.

Richard Graham, Chair, Northern Ireland Dental Practice Committee commented: 'Dental practice ventilation has been one of the key barriers to increasing patient throughput during this pandemic. We therefore welcome the Department of Health's willingness to listen to our concerns and the expediency with which it secured this additional funding.

'We look forward to working with the Department in the months ahead to address other barriers to increasing patient throughput such as PPE funding and the physically exhausting experience of wearing Level 2 PPE for extended periods.

'We must all work together to secure the sustainability of Health Service dentistry throughout this COVID-19 pandemic and beyond.' 